\title{
Evaluation of Fluoride-Induced Oxidative Stress in Rat Brain: A Multigeneration Study
}

\author{
P. Mahaboob Basha $\cdot$ Puja Rai $\cdot$ Shabana Begum
}

Received: 4 May 2010 / Accepted: 14 July 2010 /

Published online: 24 July 2010

(C) Springer Science+Business Media, LLC 2010

\begin{abstract}
Multigenerational evaluation was made in rats on exposure to high fluoride (100 and $200 \mathrm{ppm}$ ) to assess neurotoxic potential of fluoride in discrete areas of the brain in terms of lipid peroxidation and the activity of antioxidant enzyme system. The rats were given fluoride through drinking water (100 and $200 \mathrm{ppm}$ ) and maintained subsequently for three generations. Fluoride treatment significantly increased the lipid peroxidation and decreased the activity of antioxidant enzymes viz, catalase, superoxide dismutase, glutathione peroxidase, glutathione S-transferase, and glutathione level in first-generation rats and these alterations were more pronounced in the subsequent second and thirdgeneration rats in both the doses tested. Decreased feed and water consumption, litter size and organ (brain) somatic index, marginal drop in body growth rate and mortality were observed in all three generations. Decreased antioxidant enzyme activity and increased malondialdehyde levels found in the present study might be related to oxidative damage that occurs variably in discrete regions of the brain. Results of this study can be taken as an index of neurotoxicity in rats exposed to water fluoridation over several generations.
\end{abstract}

Keywords Fluoride toxicity · Multigeneration · Oxidative stress ·

Discrete brain regions $\cdot$ Rats

\section{Introduction}

Fluorosis is most severe and widespread in India as well as in China. UNICEF estimates fluorosis to be endemic in at least 25 countries across the globe [1]; as a result, fluoride acts as a potential environmental health hazard that may damage many physiological systems of human and animals to induce changes in organ systems like liver, brain, kidney, and spinal

P. M. Basha $(\square) \cdot$ P. Rai

Bangalore University, Bangalore, India

e-mail: pmbashabub@rediffmail.com

S. Begum

Maharanis Science College for Women, Bangalore, India 
cord $[2,3]$. The total number of people affected is unknown but a conservative estimate of affected individuals would number in the tens of millions due to consumption of fluoride over generations in endemic areas [1]. Earlier studies have documented considerable evidence of direct toxic effects of fluoride on the brain tissue, even at levels as low as $1 \mathrm{ppm}$ fluoride in water [4] causing impairments like altered neuronal and cerebrovascular integrity [4], abnormal behavior patterns [5] and metabolic lesions in experimental animals [6-8]. Being a central nervous system toxin, fluoride also adversely induces neuron apoptosis [9], decreased cerebral functions, and impairment in the memory-learning abilities [3, 10]. Significant increase in homovanillic acid in the hypothalamus of the mice administered with 5 ppm of sodium fluoride was indicated [11] as well as DNA damage in brain cells of rat and histopathological changes in the brain of offspring' exposed to high fluoride was observed [12]. Our previous work indicated fluoride's ability to cross the placental barrier and enter the fetus and thereby accumulate in discrete regions of brain tissues and cause toxic impact on the development [13]. Although fluoride causes injury to the central nervous system by several mechanisms, of particular interest is its ability to cause oxidative damage and various authors have reported on the fluoride-induced oxidative stress, but there is a dearth of information in brain regional specific studies in multigeneration animals exposed to fluoride, hence this study was undertaken.

\section{Materials and Methods}

\section{Chemicals}

5,5-dithio-bis-2-nitrobenzoic acid (DTNB; Ellman's reagent), epinephrine, and glutathione were procured from Sigma-Aldrich Ltd., standards and other chemicals (AR grade) from Merck Ltd.

\section{Preparation of Fluoride Water}

A stock of 1,000 ppm sodium fluoride solution was prepared by dissolving $2.21 \mathrm{~g}$ of sodium fluoride in $1 \mathrm{~L}$ of tap water. To prepare 100 and $200 \mathrm{ppm}$ fluoride water, 100 and $200 \mathrm{~mL}$ of the stock solution was taken and made up to 1 Lwith tap water, respectively.

Animals

The protocol of this study was approved by the Institutional Animal Ethics Committee, Bangalore University, Bangalore, India. Healthy adult female albino Wistar rats, weighing 170-200 g and male rats weighing 200-250 g were procured from Sri Raghavendra Enterprises, Bangalore, acclimated for a week, and maintained at room temperature of $25 \pm 2{ }^{\circ} \mathrm{C}$ with 12 -h dark-light cycle. Animals were fed with standard rodent diet (Amruth feeds, India). There was no water and light restriction throughout the experiment.

\section{Experimental Design}

For a multigenerational study 40 adult Wistar rats $\left(\mathrm{F}_{0}\right)$ were selected and maintained in ten cages, each of which consisted of three females (weighing 170-200 g) and one male (weighing 200-250 g). The rats of each group were housed in a cage for a night and then the following day the vaginal plugs of the females of each group were examined to confirm 
pregnancy. Only 24 of 30 females were identified as pregnant, and these 24 pregnant females were selected to obtain the first-generation rats $\left(\mathrm{F}_{1}\right)$. These 24 pregnant rats were then divided into three groups of eight rats each and treated with the following doses of fluoride: group I (control) was provided with tap water ad libitum $(<1 \mathrm{ppm} \mathrm{F}$ ), group II was given 100 ppm fluoride in drinking water, group III was given 200 ppm fluoride in drinking water during the gestation period.

After the gestation period of $21 \pm 2$ days there were 199 pups in the litters of the three treatment groups. The mothers received fluoridated water during the lactation period (21 days). Some of the pups from the first generation of all the three treatment groups were sacrificed on 21 st postpartum day for the biochemical estimations. The remaining pups had free access to the fluoridated water until they became mature. During all these periods, seven pups died and 192 survived. Group I (control) had no mortality (all 82 pups survived), group II had 57 surviving pups, and in group III, 53 pups survived.

Only certain numbers of adult rats from the first generation in each group were selected to continue the study on the basis of being healthy. Hence group I consisted of six females and two males, II and III consisted of nine females and three males in each group. Twentytwo female rats were identified as pregnant and were subjected to the same experimental procedure as above. The pups so obtained were considered as the second-generation rats $\left(\mathrm{F}_{2}\right)$. There were 168 pups in the litters of the three treatment groups. Some of the pups from this second generation of all the three treatment groups were sacrificed on 21 st postpartum day for the biochemical estimations. The remaining pups had free access to the fluoridated water until they became mature. During this period, 18 pups died and 150 survived. Group I (control) had no mortality (all 55 pups survived), group II had 50 surviving pups, and in group III, 45 pups survived.

To obtain the third-generation rats $\left(\mathrm{F}_{3}\right)$ six females and two males from group $\mathrm{I}$, and nine females and three males each from group II and III were selected. Twenty-two female rats were identified as pregnant and were subjected to the same experimental procedure as above. There were 163 pups in the litters of the three treatment groups. During all these periods, 21 pups died and 142 rats survived. Group I (control) had no mortality (all 56 pups survived), group II had 47 surviving pups, and in group III 39 pups survived. Some of the pups from this third-generation of all the three treatment groups were sacrificed on 21 st postpartum day and discrete regions of the brain viz. cerebral cortex, cerebellum, medulla, and hippocampus were separated out, homogenized, and used for biochemical estimations.

\section{In Vivo Biochemical Assays}

Lipid Peroxidation Lipid peroxidation (LPO) product was estimated by measurement of thiobarbituric acid reactive substances (TBARS) using the method of Niehaus and Samuelsson [14]. The pink chromogen produced by the reaction of thiobarbituric acid with malondialdehyde, a secondary product of lipid peroxidation was estimated at $535 \mathrm{~nm}$.

Catalase (EC 1.11.1.6) Catalase (CAT) activity was measured as described by Aebi [15], the decomposition of hydrogen peroxide was monitored by measuring the decrease in absorbance at $240 \mathrm{~nm}$.

Superoxide Dismutase (EC 1.15.1.1.) Superoxide Dismutase (SOD) activity was assayed by measuring the inhibition of epinephrine auto-oxidation as described by Misra and Fridovich [16]. 
Glutathione Peroxidase Activity (EC 1.11.1.9) Glutathione Peroxidase Activity (GSH-Px) activity was estimated by measuring the oxidation of NADPH as described by Lawrence and Burk [17].

Glutathione-S-transferase (EC 2.5.1.18) Glutathione-S-transferase (GST) activity was estimated by the method of Habig et al. [18] by following the increase in absorbance at $340 \mathrm{~nm}$ using 1-chloro-2,4-dinitrobenze (CDNB) as substrate.

Reduced Glutathione Reduced glutathione (GSH) content was determined by the method of Ellman [19] based on the development of a yellow color while adding DTNB to compounds containing sulphydryl groups.

Protein Assay Protein content was estimated by the method of Lowry et al. [20] using bovine serum albumin as standard.

Simultaneously, feed and water consumption of pregnant rats, litter size, mortality of pups, organ (brain) somatic index were noted on 21st postpartum day and body weight of pups from birth until 12 weeks were recorded weekly for all the three generations in control and fluoride-treated rats.

Statistical Analysis

Statistical analysis was done by one-way analyses of variance with Duncan's multiple range test (DMRT) post hoc at $P<0.05$ level of significance by using SPSS software (17.0 version).

\section{Results}

The feed and water consumption in fluoride-treated pregnant rats decreased considerably in all the three generations studied in both the doses tested when compared to control (Table 1). Although there was not much difference in the litter size between the three generations on fluoride exposure (Table 2), a slight decrease in body weight of pups at parturition was observed when compared to control and significant decrease in organ (brain) somatic index at 21st postpartum day in all three generations was observed (Table 3). Mortality was observed in all three generations (Table 2) and a marginal drop in the growth rate of pups from birth until maturity was observed in fluoride exposed animals when compared to control (Fig. 1).

\section{Lipid Peroxidation}

To evaluate the possible consequences of oxidative stress in the brain, lipid peroxidation (LPO) in terms of malondialdehyde (MDA) level was measured in different regions of the brain (Table 4). A significant increase in MDA level in the cerebral cortex (1.4-fold), cerebellum (1.5-fold), medulla (1.4-fold), and hippocampus (2.0-fold) with 100-ppm dose of fluoride, and cerebral cortex (1.4-fold), cerebellum (1.3-fold), medulla (1.1-fold), and hippocampus (1.4-fold) with 200-ppm dose of fluoride was observed in second-generation rats when compared to the first. Comparatively, more increase in MDA levels in thirdgeneration rats was observed in comparison to the first with significant changes in cerebral cortex (3.4-fold), cerebellum (1.9-fold), medulla (2.1-fold), and hippocampus (3.1-fold) 
Table 1 Average Feed and Water Consumption by Control and Fluoride Exposed Pregnant Rats in Three Generations

\begin{tabular}{llcc}
\hline Generations & Treatments & Parameters & \\
\cline { 3 - 4 } & & $\begin{array}{c}\text { Average feed consumption } \\
\text { (g feed/animal/day) }\end{array}$ & $\begin{array}{c}\text { Average water intake } \\
\text { (mL water/animal/day) }\end{array}$ \\
\hline $\mathrm{F}_{1}$ & Control & $20.45 \pm 0.46 \mathrm{e}$ & $24.88 \pm 0.58 \mathrm{f}$ \\
& 100 ppm F & $17.15 \pm 0.47 \mathrm{~d}$ & $20.23 \pm 0.38 \mathrm{e}$ \\
& & $(-16.14)$ & $(-18.69)$ \\
$\mathrm{F}_{2}$ & $16.75 \pm 0.41 \mathrm{~d}$ & $19.7 \pm 0.24 \mathrm{~d}, \mathrm{e}$ \\
& & $(-18.09)$ & $(-20.82)$ \\
& & $20.32 \pm 0.50 \mathrm{e}$ & $24.14 \pm 0.60 \mathrm{f}$ \\
& & $16.15 \pm 0.47 \mathrm{c}, \mathrm{d}$ & $18.93 \pm 0.44 \mathrm{c}, \mathrm{d}$ \\
& & $(-20.52)$ & $(-21.58)$ \\
$\mathrm{F}_{3}$ & $100 \mathrm{ppm} \mathrm{F} \mathrm{F}$ & $15.25 \pm 0.57 \mathrm{~b}, \mathrm{c}$ & $(-25.44)$ \\
& & $(-24.95)$ & $24.36 \pm 0.41 \mathrm{~b}, \mathrm{c}$ \\
& & $20.53 \pm 0.43 \mathrm{e}$ & $17.15 \pm 0.40 \mathrm{a}, \mathrm{b}$
\end{tabular}

Values are mean $\pm \mathrm{SE}$ of six animals

Mean values of different letters (a, b, c, d, e, f) within each row are statistically significant $(P<0.05)$ as determined by DMRT

Values in parenthesis indicate percentage change, '+' sign indicate increase, '-' sign indicate decrease over control

$F_{1}$ represents first generation, $F_{2}$ represents second generation, $F_{3}$ represents third generation

with 100-ppm dose of fluoride, and cerebral cortex (2.0-fold), cerebellum (1.9-fold), medulla (2.2-fold), and hippocampus (2.8-fold) with 200-ppm dose of fluoride. Similarly, when second-generation rats were compared with third generation, significant increase in MDA level in cerebral cortex (2.4-fold), cerebellum (1.3-fold), medulla (1.6-fold), and hippocampus (1.5-fold) was noticed with 100 -ppm dose of fluoride, and an increase in cerebral cortex (1.4-fold), cerebellum (1.4-fold), medulla (2.0-fold), and hippocampus (2.1-fold) with 200-ppm dose of fluoride was observed.

\section{Catalase Activity}

CAT activity was significantly decreased in cerebral cortex (1.2-fold), cerebellum (1.2-fold), medulla (1.4-fold), and hippocampus (1.1-fold) with 100-ppm dose of fluoride, and cerebral cortex (1.4-fold), cerebellum (1.2-fold), medulla (1.3-fold), and hippocampus (1.1-fold) with 200 -ppm dose of fluoride in second-generation rats when compared to the first. When thirdgeneration rats were compared with first generation, a significant decrease in CAT activity was observed in cerebral cortex (1.9-fold), cerebellum (1.3-fold), medulla (1.6-fold), and hippocampus (1.3-fold) with 100-ppm dose of fluoride, and cerebral cortex (2.0-fold), cerebellum (1.2-fold), medulla (1.6-fold), and hippocampus (1.4-fold) with 200-ppm dose of fluoride. Similarly, a significant decrease between second and third generation 
Table 2 Multigenerational Effects of Fluoride on Litter Size and Percentage Mortality in Rats

\begin{tabular}{|c|c|c|c|}
\hline \multirow[t]{2}{*}{ Generations } & \multirow[t]{2}{*}{ Treatments } & \multicolumn{2}{|l|}{ Parameters } \\
\hline & & Mean litter size & Percent mortality $(\%)$ \\
\hline \multirow[t]{5}{*}{$\mathrm{F}_{1}$} & Control & $10.25 \pm 0.96 \mathrm{c}$ & 0 \\
\hline & $100 \mathrm{ppm} \mathrm{F}$ & $7.5 \pm 0.8 \mathrm{a}, \mathrm{b}$ & 5 \\
\hline & & $(-26.83)$ & \\
\hline & $200 \mathrm{ppm} \mathrm{F}$ & $7.13 \pm 0.92 \mathrm{a}, \mathrm{b}$ & 7.02 \\
\hline & & $(-30.44)$ & \\
\hline \multirow[t]{5}{*}{$\mathrm{F}_{2}$} & Control & $9.17 \pm 0.48 b, c$ & 0 \\
\hline & 100 ppm F & $7.25 \pm 1.01 \mathrm{a}, \mathrm{b}$ & 13.79 \\
\hline & & $(-20.94)$ & \\
\hline & 200 ppm F & $6.88 \pm 0.52 \mathrm{a}, \mathrm{b}$ & 18.18 \\
\hline & & $(-24.97)$ & \\
\hline \multirow[t]{5}{*}{$\mathrm{F}_{3}$} & Control & $9.33 \pm 0.49 b, c$ & 0 \\
\hline & 100 ppm F & $7.13 \pm 0.64 \mathrm{a}, \mathrm{b}$ & 17.54 \\
\hline & & $(-23.58)$ & \\
\hline & $200 \mathrm{ppm} \mathrm{F}$ & $6.25 \pm 0.59 \mathrm{a}$ & 22 \\
\hline & & $(-33.01)$ & \\
\hline
\end{tabular}

Values are mean \pm SE of six animals

Mean values of different letters $(a, b, c)$ within each row are statistically significant $(P<0.05)$ as determined by DMRT

Values in parenthesis indicate percentage change, '+' sign indicate increase, '-' sign indicate decrease over control

$F_{1}$ represents first generation, $F_{2}$ represents second generation, $F_{3}$ represents third generation

were observed in cerebral cortex (1.6-fold), cerebellum (1.6-fold), medulla (1.1-fold), hippocampus (1.2-fold) with 100-ppm dose of fluoride, and cerebral cortex (1.4-fold), cerebellum (1.1-fold), medulla (1.2-fold), and hippocampus (1.2-fold) with 200-ppm dose of fluoride was observed.

\section{Superoxide Dismutase Activity}

The activity of SOD was observed to be significantly decreased in cerebral cortex (1.2-fold), cerebellum (1.1-fold), medulla (1.1-fold), and hippocampus (1.1-fold) with 100-ppm dose of fluoride and cerebral cortex (1.2-fold), cerebellum (1.1-fold), medulla (1.1-fold), and hippocampus (1.1-fold) with 200-ppm dose of fluoride in second-generation rats when compared to the first. Significant decrease was observed in cerebral cortex (1.4-fold), cerebellum (1.3-fold), medulla (1.2-fold), and hippocampus (1.2-fold) with 100-ppm dose of fluoride and cerebral cortex (1.5-fold), cerebellum (1.3-fold), medulla (1.3-fold), and hippocampus (1.2-fold) with 200-ppm dose of fluoride was observed when third-generation rats were compared with first generation. Between second- and third-generation rats significant decrease were observed in cerebral cortex (1.3-fold), cerebellum (1.1-fold), medulla (1.1-fold), and hippocampus (1.1-fold) with 100-ppm dose of fluoride and cerebral cortex (1.2-fold), cerebellum (1.1-fold), medulla (1.2-fold), and hippocampus (1.1-fold) with 200-ppm dose of fluoride. 
Table 3 Multigenerational Effects of Fluoride on Body Weight and Organ (Brain) Somatic Index in Rats

\begin{tabular}{llcc}
\hline Generations & Treatments & Parameters & \\
\cline { 3 - 3 } & & Body weight at birth $(\mathrm{g})$ & Organ (brain) somatic index \\
\hline $\mathrm{F}_{1}$ & Control & $10.0 \pm .0 .002 \mathrm{c}$ & $6.46 \pm 0.08 \mathrm{~g}$ \\
& $100 \mathrm{ppm} \mathrm{F}$ & $9.0 \pm 0.012 \mathrm{~b}$ & $5.47 \pm 0.13 \mathrm{f}$ \\
& & $(-10.0)$ & $(-15.33)$ \\
$\mathrm{F}_{2}$ & $8.5 \pm 0.27 \mathrm{a}, \mathrm{b}$ & $4.91 \pm 0.04 \mathrm{e}$ \\
& & $(-15.0)$ & $(-23.99)$ \\
& & $10.1 \pm 0.005 \mathrm{c}$ & $6.34 \pm 0.11 \mathrm{~g}$ \\
& & $8.33 \pm 0.21 \mathrm{a}, \mathrm{b}$ & $4.42 \pm 0.08 \mathrm{~d}$ \\
& & $(-17.53)$ & $(-30.28)$ \\
$\mathrm{F}_{3}$ & $8.0 \pm 0.22 \mathrm{a}$ & $4.17 \pm 0.02 \mathrm{c}$ \\
& & $(-20.79)$ & $(-34.23)$ \\
& & $10.1 \pm 0.01 \mathrm{c}$ & $6.43 \pm 0.10 \mathrm{~g}$ \\
& & $8.13 \pm 0.13 \mathrm{a}$ & $3.75 \pm 0.04 \mathrm{~b}$
\end{tabular}

Values are mean \pm SE of six animals

Mean values of different letters (a, b, c, d, e, f, g) within each row are statistically significant $(P<0.05)$ as determined by DMRT

Values in parenthesis indicate percentage change, '+' sign indicate increase, '-' sign indicate decrease over control

$F_{1}$ represents first generation, $F_{2}$ represents second generation, $F_{3}$ represents third generation

\section{Glutathione Peroxidase Activity}

The activity of GSH-Px was observed to be significantly decreased in the cerebral cortex (2.3-fold), cerebellum (1.2-fold), medulla (1.6-fold), and hippocampus (1.1-fold) with $100-p p m$ dose of fluoride and cerebral cortex (1.8-fold), cerebellum (1.2-fold), medulla (2.1-fold), and hippocampus (1.9-fold) with 200-ppm dose of fluoride in second-generation rats when compared to first generation. When third-generation rats were compared with first generation, significant decrease in GSH-Px activity was observed in cerebral cortex (2.5-fold), cerebellum (1.3-fold), medulla (2.2-fold), hippocampus (1.1-fold) with 100-ppm dose of fluoride and cerebral cortex (4.5-fold), cerebellum (2.4-fold), medulla (2.5-fold), and hippocampus (2.2-fold) with 200-ppm dose of fluoride. Between second generation and third-generation rats decreased activity was observed in cerebral cortex (1.1-fold), and cerebellum (1.1-fold), medulla (1.4-fold), and hippocampus (1.1-fold) with 100-ppm dose of fluoride and cerebral cortex (2.5-fold), cerebellum (2.0-fold), medulla (1.2-fold), and hippocampus (1.2-fold) with 200-ppm dose of fluoride.

\section{Glutathione S-transferase Activity}

On examination of differences between first- and second-generation rats, glutathione-Stransferase activity was significantly decreased in cerebral cortex (1.3-fold), cerebellum 


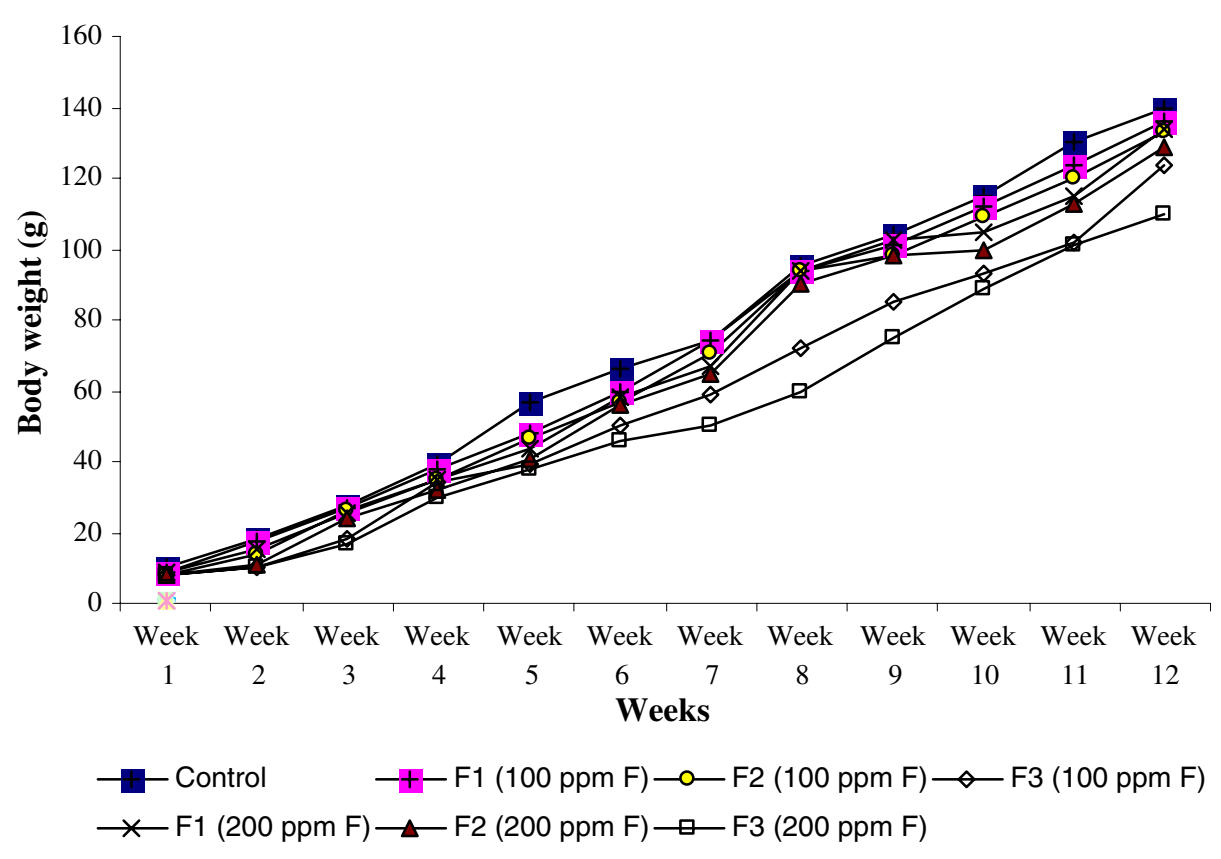

Fig. 1 Mean body weight of pups from birth until 12 weeks in multigeneration rats

(1.4-fold), medulla (1.2-fold), and hippocampus (1.2-fold) regions with 100-ppm dose of fluoride and cerebral cortex (1.4-fold), cerebellum (1.4-fold), medulla (1.2-fold), and hippocampus (1.2-fold) with 200-ppm dose of fluoride. Between third and first-generation rats, a marked decrease was observed in cerebral cortex (1.6-fold), cerebellum (1.7-fold), medulla (1.5-fold), and hippocampus (1.9-fold) regions with 100-ppm dose of fluoride and cerebral cortex (2.2-fold), cerebellum (2.5-fold), medulla (1.6-fold), and hippocampus (1.8-fold) with 200-ppm dose of fluoride. Suppressed activity levels were observed in cerebral cortex (1.2-fold), cerebellum (1.2-fold), medulla (1.3-fold), and hippocampus (1.6-fold) regions with 100-ppm dose of fluoride and cerebral cortex (1.6-fold), cerebellum (1.7-fold), medulla (1.3-fold), and hippocampus (1.8-fold) with 200-ppm dose of fluoride when second-generation rats were compared with third generation.

\section{Reduced Glutathione Level}

There was a significant decline in reduced glutathione levels in cerebral cortex (1.2-fold), cerebellum (1.1-fold), medulla (1.2-fold), and hippocampus (1.1-fold) with 100-ppm dose of fluoride and cerebral cortex (1.1-fold), cerebellum (1.1-fold), medulla (1.2-fold), and hippocampus (1.1-fold) with 200-ppm dose of fluoride in second-generation rats when compared to the first. Between third and first-generation rats, a marked decrease in GSH levels were observed in cerebral cortex (1.3-fold), cerebellum (1.2-fold), medulla (1.3-fold), and hippocampus (1.2-fold) with 100-ppm dose of fluoride and cerebral cortex (1.3-fold), cerebellum (1.1-fold), medulla (1.4-fold), and hippocampus (1.3-fold) with 200-ppm dose of fluoride. Similarly, a significant decrease between second and third-generation rats was 
observed in cerebral cortex (1.1-fold), cerebellum (1.1-fold), medulla (1.1-fold), and hippocampus (1.1-fold) with 100-ppm dose of fluoride and cerebral cortex (1.1-fold), cerebellum (1.1-fold), medulla (1.1-fold), and hippocampus (1.2-fold) with 200-ppm dose of fluoride.

\section{Discussion}

Excessive ingestion of fluoride considerably decreased the animal growth symptomised by decreased appetite leading to poor growth rate as noticed in litters in all the three generations. The decrease found in body weight could be due to primary malnutrition caused by fluoride by displacing other nutrients present in the diet (minerals or elements). Secondary malnutrition may result either from maldigestion or malabsorption of nutrients caused by gastrointestinal complications. These nutritional complexities can induce deficiency virtually on all the nutrients [21]. Earlier studies have suggested fluoride to produce overt maternal toxicity, and feto-toxicity $[22,23]$ indicating that maternal toxicity affects the viability or growth of offsprings. In the present study, fluoride exposure (100 and $200 \mathrm{ppm}$ ) caused a marginal drop in the post natal growth rate and organ (brain) somatic index (Fig. 1, Table 3). Further, maternal exposure caused deficits in birth weight, and survival of pups indicating suppressed growth of offsprings. The profile analysis showed that fluoride regimen employed marginally influenced the weight of pups at birth and also their growth rate until maturity.

Although the blood-brain barrier is relatively impermeable to fluoride, it does not pose an absolute barrier and fluoride has the ability to enter the brain [24]. High fluoride exposure can result in damage to brain cells, however, because the damage is usually mild, obvious functional changes will not necessarily be readily observable [25]. Since fluoride accumulation begins in the placenta during gestation, the transfer of fluoride to fetus appears to be restricted. Furthermore, fluoride might have been transferred from dam to pups during first 3 weeks after birth when the offsprings suckle. In addition, neonatal rats absorb and retain substantially more fluoride than older animals. Our previous study reported maternal fluoride exposure to cause a remarkable increase in the level of fluoride in the discrete brain regions of the pups [13]. In this study, the exposure commences in the maternal blood, passes through the placenta to the fetus and continues during neonatal to adulthood through fluoride-containing milk and drinking water, respectively. Moreover, the immaturity of excretory system favors the accumulation when the progeny continues with the exposure of fluoride for several generations and the functional areas of the brain may become more sensitive for accumulation. The results further suggest that high fluoride intake over several generations has the ability to elevate lipid peroxidation and alter the antioxidant enzyme system, thereby leading to increased oxidative stress and therefore become a mediating factor in the pathogenesis of fluoride toxicity. Meral et al. [26] reported a significant increase in TBARS level in the testes of both first- and second-generation rats when compared to control, and the TBARS levels of second-generation rats were significantly higher than those of first-generation rats. Aydin et al. [27] reported a significant increase in TBARS levels in lung tissues in groups treated with 50 and $100 \mathrm{mg} / \mathrm{L}$ fluoride over several generations. Similarly, Karaoz et al. [28] showed that chronic fluorosis can lead to enhanced lipid peroxidation and kidney tissue alterations in first- and secondgeneration rats.

In this study, effect of fluoride exposure during gestation and post gestation periods were studied to analyze oxidative stress parameters in different brain regions for three 


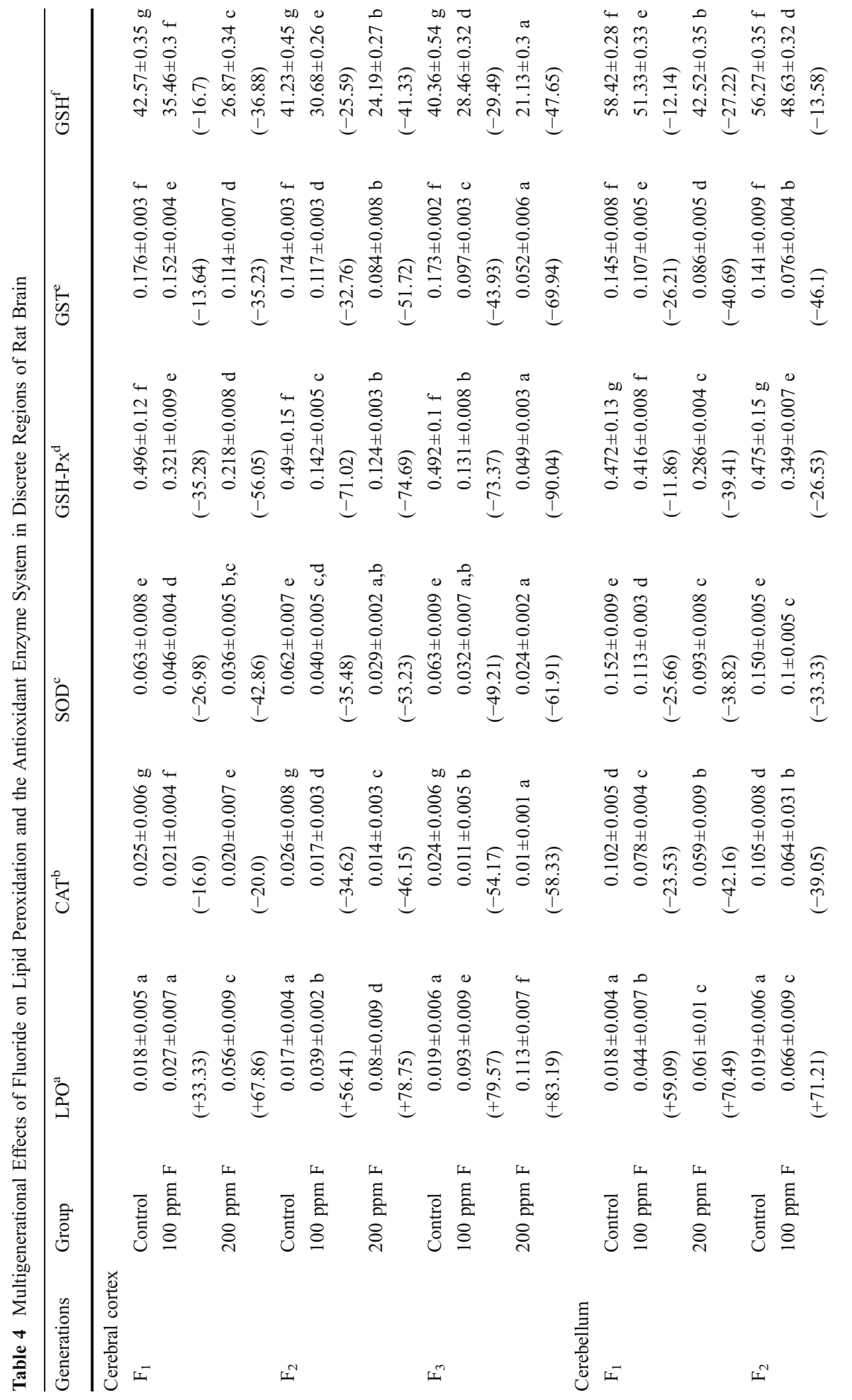




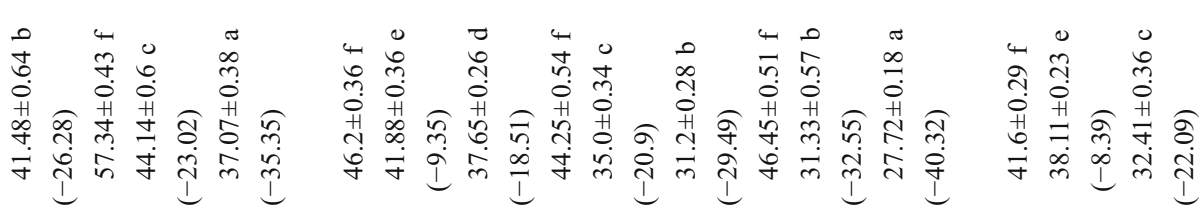

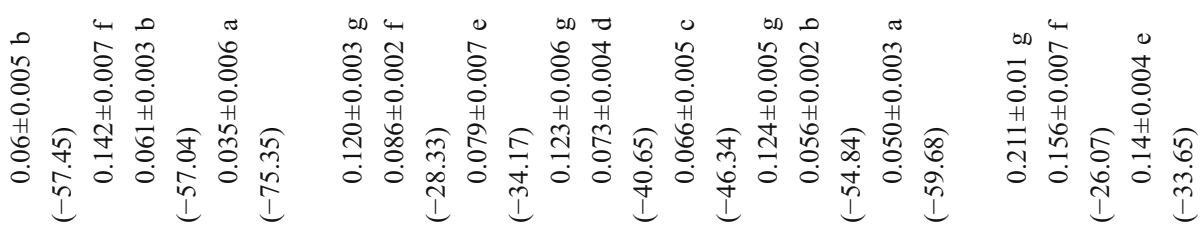

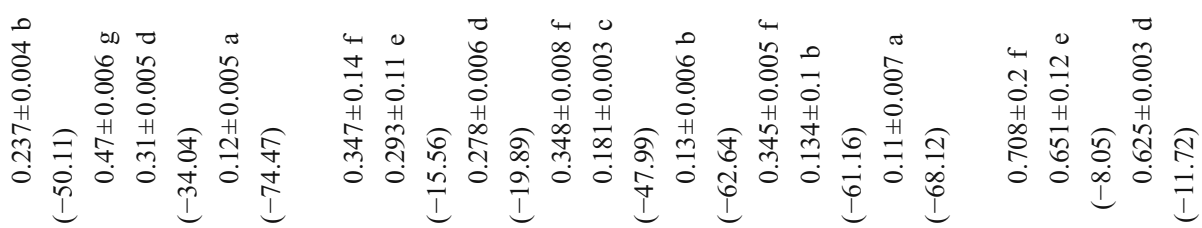

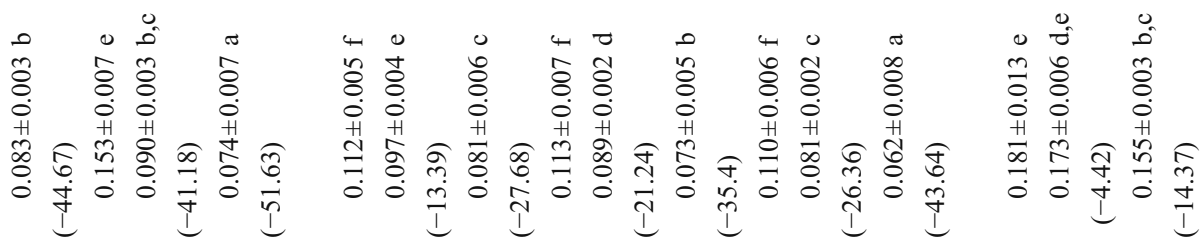

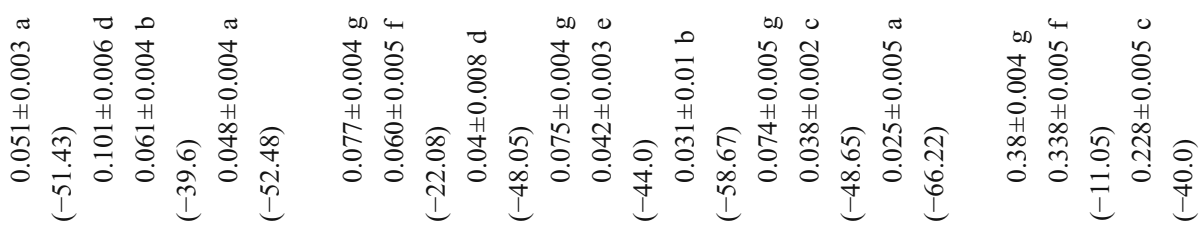

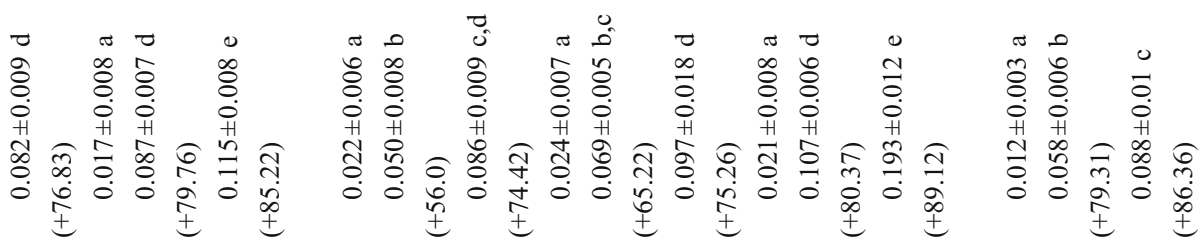

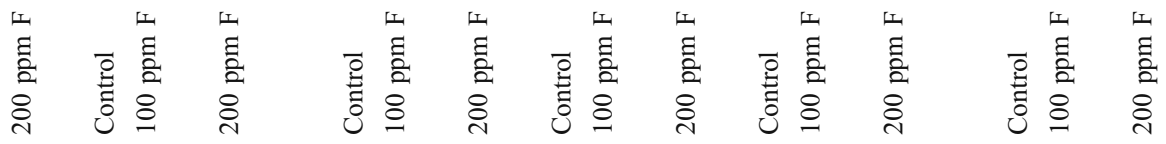<smiles></smiles> 


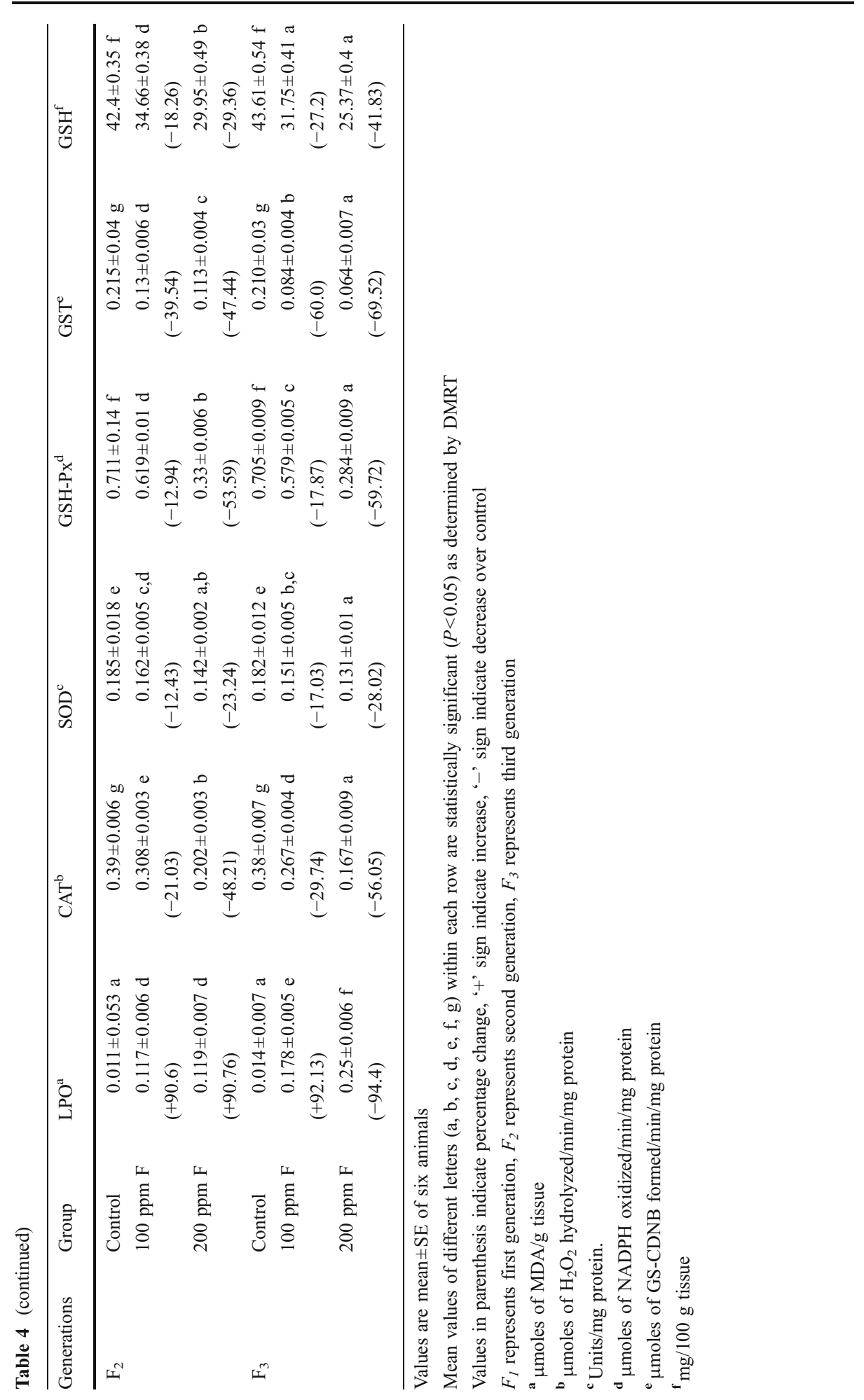


generations and we observed a greater sensitivity to fluoride in second- and third-generation rats compared to first generation. The activities of CAT, SOD, GSH-Px, GST, and GSH were decreased, while the level of MDA was increased in the experimental rats exposed to high fluoride (100 and $200 \mathrm{ppm}$ ) as compared to the control group. The observed increase in lipid peroxidation in discrete brain regions, as determined by the measurement of MDA, is also in agreement with previous studies [29-32]. Therefore, in line with the results of earlier reports, it could be suggested that the elevated lipid peroxidation in fluorosis may result from the decreased activities of antioxidant enzymes [32-34]. There have been many reports suggesting that free radicals play an aberrant role in the mechanism of fluoride toxicity [35-37]. High lipid content in the brain is responsible for the vulnerability of these tissues to oxidative stress. This is because peroxidative damage of membrane lipids leads to many damages in a cell such as decreased membrane fluidity, elevated sensitivity to oxidant stress and changes in enzyme activities [38]. Therefore, the consequences of chronic fluoride exposure could be attributed to induce lipid peroxidation. Decreased activity of CAT and SOD in the discrete regions of the brain may be in response to increased production of $\mathrm{H}_{2} \mathrm{O}_{2}$ and $\mathrm{O}_{2}$. Further decreased activity of SOD may be due to competitive inhibition by the fluoride ion for binding to the active site of copper [39], iron or manganese with SOD, or due to a direct action of fluoride on the enzymes (competitive inhibition) as well as free radicals rather due to increased free radicals alone [40]. The traditional role of GSH is that of a free radical scavenger; the ability of GSH to nonenzymatically scavenge both singlet oxygen and $\mathrm{OH}$-radical provides the first line of antioxidant defense. The decrease found in the levels of GSH in fluoride-treated rats may be due to increased utilization of GSH by glutathione peroxidase in detoxification of $\mathrm{H}_{2} \mathrm{O}_{2}$ generated by fluoride-induced oxidative stress [41]. The reduction in GSH might be attributed to the fact that fluoride creates an imbalance between the pro-oxidant and the antioxidants inside the body due to which it is unable to withstand the stress leading to the reduction in GSH level [42]. Mittal and Flora [43, 44] reported that fluoride exposure decreases the level of GSH and inhibits various enzymes which require GSH as a cofactor. Depletion of GSH is related to the decreased antioxidant status and the overproduction of reactive oxygen species (ROS). GST binds electrophilic moieties and various toxicants and drugs with glutathione and offers a powerful mechanism for detoxification [45].

Since the brain exhibits considerable regional heterogeneity, the effect of high fluoride exposure in discrete brain regions was examined for three generations. In this study, chronic fluoride exposure caused significant increase in MDA levels in discrete brain regions of first-generation rats, which were more pronounced in the subsequent second and third generations. Differences in susceptibilities of different regions to fluoride may be due to differences in capabilities of different regions to combat oxidative stress, perhaps the distribution of enzymatic and non-enzymatic antioxidants are not uniform in heterogenous brain regions [46]. The medulla and hippocampus appear to be more vulnerable to ROS injury due to inherent low levels of GSH compared to cerebrum and cerebellum [46]. Moreover, the difference in ROS burden in various brain regions is due to well known heterogeneity and metabolic compartmentalization of the brain mitochondria [47]. In this study, the differential sensitivity found in second and third-generation rats on fluoride exposure in different brain regions may be due to preferential fluoride accumulation and also due to altered biochemical and cellular processes in particular regions. The accumulated fluoride acts as a crucial factor in altering cellular processes via other vital elemental alterations that in turn alter cellular redox homeostasis. 
From this study, it is concluded that the multigenerational exposure to fluoride via drinking water cause oxidative stress in discrete regions of rat brain in all the three generations and these alterations were more pronounced in second and third-generation rats compared to the first. Further fluoride exposure during pregnancy, development, and thereafter can adversely affect the outcome of offsprings.

Acknowledgement University Grants Commission, New Delhi, India for research grants (F.No. 31-220/ 2005 (SR) dated 31-03-2006 \& MRP(S)/139/08-09/KABA027/UGC/SWRO).

\section{References}

1. Fluoride Action Network. (May 2004) http://www.fluoridealert.org/fluorosis-india.htm/

2. Guan ZZ, Wang YN, Xiao KQ, Dai DY, Chen YH, Liu JL, Sindelar P, Dallner G (1998) Influence of chronic fluorosis on membrane lipids in rat brain. Neurotoxicol Teratol 20:537-542

3. Wang J, Ge Y, Ning H, Wang S (2004) Effects of high fluoride and low iodine on oxidative stress and antioxidant defense of the brain in offspring rats. Fluoride 37:264-270

4. Varner JA, Jensen KF, Horvath W, Isaacson RL (1998) Chronic administration of aluminum-fluoride or sodium-fluoride to rats in drinking water: alterations in neuronal and cerebrovascular integrity. Brain Res 784:284-298

5. Mullenix PJ, Denbesten PK, Schunior A, Kernan WJ (1995) Neurotoxicity of sodium fluoride in rats. Neurotoxicol Teratol 17:169-177

6. Shashi A (1992) Studies on alterations in brain lipid metabolism following experimental fluorosis. Fluoride 25:77-84

7. Shashi A, Singh JP, Thapar SP (1994) Effect of long-term administration of fluoride on levels of proteins, free amino acids and RNA in rabbit brain. Fluoride 27:155-159

8. Vani ML, Reddy KP (2000) Effects of fluoride accumulation on some enzymes of brain and gastonemius muscle of mice. Fluoride 33:17-26

9. Lu XH, Li GS, Sun B (2000) Study of the mechanism of neuron apoptosis in rats from the chronic fluorosis. Chinese J Endemiol 19:96-98

10. Wang YN, Xiao KQ, Liu JL, Dallner G, Guan ZZ (2004) Effects of long term fluoride exposure on lipid composition in rat liver. Toxicology 146:161-169

11. Tsunoda M, Aizawa Y, Nakano K, Liu Y, Horiuchi T, Ltai K, Tsunoda H (2005) Changes in fluoride levels in the liver, kidney and brain and in neurotransmitters of mice after sub acute administration of fluoride. Fluoride 38:284-292

12. Ge Y, Ning H, Wang S, Wang J (2005) Effects of high fluoride and low iodine on brain histopathology in offspring rats. Fluoride 38:127-132

13. Madhusudhan N, Mahaboob BP (2010) Effect of maternal exposure of fluoride on biometals and oxidative stress parameters in developing CNS of rat. Biol Trace Elem Res 133:71-82

14. Niehaus WG Jr, Samuelsson B (1968) Formation of malonaldehyde from phospholipid arachidonate during microsomal lipid peroxidation. Eur J Biochem 6:126-130

15. Aebi H (1984) Catalase in vitro. Methods Enzymol 105:121-126

16. Misra HP, Fridovich I (1972) The role of superoxide anion in the autoxidation of epinephrine and a simple assay for superoxide dismutase. J Biol Chem 247:3170-3175

17. Lawrence RA, Burk RF (1976) Glutathione peroxidase activity in selenium-deficient rat liver. Biochem Biophys Res Commun 71:952-958

18. Habig WH, Pabst MJ, Jakoby WB (1974) Glutathione S-transferases. The first enzymatic step in mercapturic acid formation. J Biol Chem 249:7130-7139

19. Ellman GL (1959) Tissue sulfhydryl groups. Arch Biochem Biophys 82:70-77

20. Lowry OH, Rosebrough NJ, Farr AL, Randall RJ (1951) Protein measurement with the Folin phenol reagent. J Biol Chem 193:265-275

21. Lieber CS, DeCarli LM (1991) Hepatotoxicity of ethanol. J Hepatol 12:394-401

22. Mona H, Mai ED (2006) Fetotoxicity of fluoride in rats and the protective action of some antioxidants. Fluoride 39:202-210

23. Al-Hiyasat AS, Elbitieha AM, Darmani H (2000) Reproductive toxic effects of ingestion of sodium fluoride in female rats. Fluoride 33:79-84

24. Spittle B (1994) Psychopharmacology of fluoride: a review. Int Clin Psychopharmacol 9:79-82 
25. Jing L, Li Y, Qing-Liang S, Chun-Yan W (2008) Effects of high fluoride level on neonatal neurobehavioral development. Fluoride 41:165-170

26. Meral O, Ahmet K, Erdal K, Hakan D, Emin S, Fatih G (2007) Effect of long-term fluoride exposure on lipid preoxidation and histology of testes in first and second generation rats. Biol Trace Elem Res 118:260-268

27. Aydin G, Cicek E, Akdogan M, Gokalp O (2003) Histopathological and biochemical changes in lung tissues of rats following administration of fluoride over several generations. J Appl Toxicol 23:437-446

28. Karaoz E, Oncu M, Gulle K, Kanter M, Gultekin F, Karaoz S, Mumcu E (2004) Effect of chronic fluorosis on lipid peroxidation and histology of kidney tissues in first- and second-generation rats. Biol Trace Elem Res 102:199-208

29. Sharma A, Chinoy NJ (1998) Role of free radicals in fluoride-induced toxicity in liver and kidney of mice and its reversal. Fluoride 31:S26

30. Patel D, Chinoy NJ (1998) Influence of fluoride on biological free radical reactions in ovary of mice and its reversal. Fluoride 31:S27

31. Chinoy NJ, Patel D (1998) Influence of fluoride on biological free radical reactions in ovary of mice and its reversal. Environ Sci 6:171-184

32. Sun G, Qiu L, Ding G, Qian C, Zheng Q (1998) Effects of $\beta$-carotene and SOD on lipid peroxidation induced by fluoride: an experimental study. Fluoride 31:S29

33. Shanthakumari D, Srinivasalu S, Subramanian S (2004) Effect of fluoride intoxication on lipidperoxidation and antioxidant status in experimental rats. Toxicology 204:219-228

34. Venkataraman P, Muthuvel R, Krishnamoorthy G, Arunkumar A, Sridhar M, Srinivasan N, Balasubramanian K, Aruldhas MM, Arunakaran J (2007) PCB (Aroclor 1254) enhances oxidative damage in rat brain regions: protective role of ascorbic acid. Neurotoxicology 28:490-498

35. Shivarajashankara YM, Shivashankara AR, Bhat PG, Rao SH (2003) Lipid peroxidation and antioxidant systems in the blood of young rats subjected to chronic fluoride toxicity. Indian J Exp Biol 41:857-860

36. Jeji J, Sharma R, Jolly SS, Pamnani S (1985) Implication of glutathione in endemic fluorosis. Fluoride 18:117-119

37. Wei ZD, Li F, Zhou L, Chen X, Dai G (1995) Studies on fluoride-aluminium combined toxicosis. Fluoride 28:37-38

38. O'Donnell E, Lynch MA (1998) Dietary antioxidant supplementation reverses age-related neuronal changes. Neurobiol Ageing 19:461-467

39. Lawson PB, Yu MH (2003) Fluoride inhibition of superoxide dismutase (SOD) from the earthworm Eisenia fetida. Fluoride 36:143-151

40. Chlubek D, Grucka-Mamczar E, Birkner E, Polaniak R, Stawiarska-Pieta B, Duliban H (2003) Activity of pancreatic antioxidative enzymes and malondialdehyde concentrations in rats with hyperglycemia caused by fluoride intoxication. J Trace Elem Med Biol 17:57-60

41. Guney M, Oral B, Take G, Giray SG, Mungan T (2007) Effect of fluoride intoxication on endometrial apoptosis and lipid peroxidation in rats: role of vitamins $\mathrm{E}$ and $\mathrm{C}$. Toxicology 231:215-223

42. Swapnila C, Vinay L, Flora SJS (2009) Fluoride-induced changes in haem biosynthesis pathway, neurological variables and tissue histopathology of rats. J Appl Toxicol 30(1):63-73

43. Mittal M, Flora SJ (2006) Effects of individual and combined exposure to sodium arsenite and sodium fluoride on tissue oxidative stress, arsenic and fluoride levels in male mice. Chem Biol Interact 162:128-139

44. Mittal M, Flora SJ (2007) Vitamin E supplementation protects oxidative stress during arsenic and fluoride antagonism in male mice. Drug Chem Toxicol 30:263-281

45. Ketterer B (1996) Detoxification reactions of glutathione and glutathione transferase Xenobiotica $16: 957-975$

46. Somani SM, Husain K (1999) Exercise and oxygen toxicity: a handbook. In: Sen CK, Packer L, Hanninen O (eds). Elsevier Sciences, New York pp 712-751

47. Villa RF, Gorini A, Zanada F, Benzi G (1986) Action of L-acetylcarnitine on different cerebral mitochondrial populations from hippocampus. Arch Int Pharmacodyn Thér 279:195-211 